\title{
Cost effectiveness of different screening strategies for gestational diabetes mellitus screening: study protocol of a randomized community non-inferiority trial
}

\author{
Fahimeh Ramezani Tehrani* and Gulf Study Cooperative Research Group
}

\begin{abstract}
Background: There is lack of ideal and comprehensive economic evaluations of various GDM strategies. The aim of this study is to the compare efficacy and cost-effectiveness of five different methods of screening for gestational diabetes mellitus (GDM).

Methods: This study is a randomized community non-inferiority trial among 30,000 pregnant women in five different geographic regions of Iran, who were randomly assigned to one of the five GDM screening methods. All first trimester pregnant women, seeking prenatal care in governmental health care systems, who met our eligibility criteria were enrolled. The criteria suggested by the International-Association-of-Diabetes-in-Pregnancy-Study-Group, the most intensive approach, were used as reference. We used the non-inferiority approach to compare less intensive strategies to the reference one. Along with routine prenatal standard care, all participants were scheduled to have two phases of GDM screening in first and second-trimester of pregnancy, based on five different pre-specified protocols. The screening protocol included fasting plasma glucose in the first trimester and either a one step or a two-step screening method in the second trimester of pregnancy. Pregnant women were classified in three groups based on the results: diagnosed with preexisting pre-gestational overt diabetes; gestational diabetes and non-GDM women. Each group received packages for standard-care and all participants were followed till delivery; pregnancy outcomes, quality of life and cost of health care were recorded in detail using specific standardized questionnaires. Primary outcomes were defined as \% birth-weight > 90th percentile and primary cesarean section. In addition, we assessed the direct health care direct and indirect costs.
\end{abstract}

Results: This study will enable us to compare the cost effectiveness of different GDM screening protocols and intervention intensity (low versus high).

Conclusion: Results which if needed, will also enable policy makers to optimize the national GMD strategy as a resource for enhancing GDM guidelines.

Trial registration Name of the registry: Iranian Registry of Clinical Trials. Trial registration number: IRCT138707081281N1. Date of registration: 2017-02-15. URL of trial registry record: https://www.irct.ir/trial/518

Keywords: Cost-effectiveness, Gestational diabetes, Screening, Perinatal outcome

*Correspondence: ramezani@endocrine.ac.ir; framezan@post.harvard.edu Reproductive Endocrinology Research Center, Research Institute

for Endocrine Sciences, Shahid Beheshti University of Medical Sciences, No 24, Parvane Street, Yaman Street, Velenjak, P.O.Box: 19395-4763, Tehran, Iran

(c) The Author(s) 2019. This article is distributed under the terms of the Creative Commons Attribution 4.0 International License (http:// creativecommons.org/licenses/by/4.0/), which permits unrestricted use, distribution, and reproduction in any medium, provided you give appropriate credit to the original author(s) and the source, provide a link to the Creative Commons license, and indicate if changes were made. The Creative Commons Public Domain Dedication waiver (http://creativecommons.org/publicdomain/zero/1.0/) applies to the data made available in this article, unless otherwise stated. 


\section{Background}

Gestational diabetes (GDM) defined as hyperglycaemia at any time during pregnancy at levels below those that occurring in overt diabetes [1]. It is one of the most common glycemic disorders during pregnancy with occurrence of $1-28 \%$ of all pregnancies [2-5], along with the increased rate of obesity and advanced maternal age is rising in prevalence [6]. It is well documented that GDM is associated with both short as well as long term higher rates of adverse feto-maternal and neonatal outcomes [7-11]. From an obstetrical perspective, evidence shows that treatment of GDM is effective in reducing the risk of many of the important adverse pregnancy outcomes [12-14].

Despite the globally accepted importance of screening for and treating GDM [13], screening strategies, testing methods and even diagnostic optimum glycemic thresholds for GDM remained much controversy for decades and no international consensus has been yet established [15]. In addition, the former screenings were mainly performed to prevent adverse maternal outcomes compared to neonatal complications. Considering this, use of different tests and criteria will impact the prevalence of women diagnosed with GDM [5], and could also impact poor pregnancy outcomes $[16,17]$. There is also much controversy about milder forms of GDM. For which, the associations of mild GDM with adverse pregnancy outcomes are not completely understood; there is ongoing debate about the benefits of treating mild GDM and the impact on health care costs [18-21].

The Hyperglycemia and Adverse Pregnancy Outcomes (HAPO) study demonstrated that hyperglycemia at levels below those previous recommended thresholds for GDM were associated with adverse maternal and neonatal outcomes; hence, the International Association of Diabetes in Pregnancy Study Group (IADPSG) introduced new cutoffs for the 2-hour (2 h) oral glucose tolerance test (OGTT) in GDM screening and diagnosis [22]. Besides, at present, a 3-h $100 \mathrm{~g}$ diagnostic test is used predominantly in the United States and some other areas, whereas much of the world uses the $75 \mathrm{~g}, 2$-h OGTT [5]. At present, there is little information regarding the sensitivity and specificity of these test, and hence the relative clinical effectiveness of the two-steps of the 1-h 50-g glucose challenge test (GCT) following 3-h $100 \mathrm{~g}$ oral glucose tolerance (OGTT) diagnostic test and the onestep OGTT approaches in the same population. However using the IADPSG criteria, two to threefold more women qualified for a diagnosis of GDM, potentially adding to the costs of care of the already large number of pregnant women [23-25].

With both increased prevalence and adopting lowering of the thresholds for diagnosis, the healthcare cost of
GDM can be expected to rise proportionately. It follows that the debate as to whether or not a benefit exists in the treatment of GDM assumes even greater importance now than in the past. However, since in most countries, resources are inevitably scarce, healthcare interventions should be evaluated for their impact the on cost as well as effectivity on clinical outcomes [26]. Moreover, while not recognizing that GDM is associated with adverse pregnancy outcomes, over-diagnosis may lead to psychological stress, unnecessary treatments and impaired quality of life [27-29].

There is lack of ideal and comprehensive economic evaluations of various GDM strategies; the majority of existing cost-effective analyses are based on decision analysis modelling not real data, limited obtained from randomized clinical trials that documented controversial results [20, 30-38]. In addition most studies have been conducted in well-developed high-income countries which obviously have more developed healthcare systems than low and middle-income countries, where gestational diabetes has the highest prevalence. According to a WHO report, global and local decision-making regarding GDM strategies are challenging due to the lack of optimum economic evaluations of various GDM screening protocols, making it difficult to validated implement any national recommendations from a health economic perspective [31]. Since resources are unavoidably scarce, national health care interventions should be assessed for their impact on costs as well as on clinical outcomes; the most highly recommended practice is that economic evaluation should be an integral part of randomized clinical trials [39]; each population needs to adopt its community specific guidelines [40].

In this ongoing randomized community-field non-inferiority trial, we aimed to compare the cost-effectiveness of five different pre-defined GDM screening protocols, both one and two step, using different fasting plasma glucose thresholds to ascertain the optimum GDM screening protocol.

\section{Materials and methods \\ Research questions and objectives}

This study is being performed to provide real data collected from an unbiased population trial for assessment of the following hypothesis: (i) the prevalence of GDM when using the less intensive GDM screening strategies is not more than obtained using the IADPSG criteria. (ii) The pre-specified primary outcomes in less intensive GDM screening strategies are not worse than those obtained using IADPSG criteria. (iv) The cost of health care using less intensive GDM screening strategies is not higher than incurred using IADPSG criteria. (v) The 
numbers needed to treat (NNT) to prevent one primary outcome in less intensive strategies are the same as those obtained using IADPSG criteria.

The cost of prevention for one primary outcome in less intensive strategies is the same as that for IADPSG criteria.

According to our research hypothesis, primary outcomes hence are: percentage of birth weight $>90$ th percentile and primary cesarean section. Secondary outcomes are prevalence of neonatal hypoglycemia, birth weight $<10$ th percentile, neonatal admission to the intensive care unit, shoulder dystocia and birth trauma including fracture of clavicle and brachial plexus injury, intrauterine fetal death, preeclampsia and preterm labor, neonatal hyperbilirubinemia and hypocalcemia. In addition, the study will assess the direct health care costs including prenatal clinic visits, obstetrician visits, endocrinologist visits, dietician visits, blood glucose monitoring equipment, laboratory test cost, pharmacotherapy, additional fetal well-being assessments and hospitalization as well as indirect cost of productivity loss and charges to the family including traveling, food substitution, mother time off paid work, and partner time off work.

\section{Overall study design}

This is a randomized community-field trial including five GDM screening strategies in a parallel group design. Recruitment of the participants took place between September 2016 and January 2019 in 1015 health centers in 25 selected cities of five provinces of Iran.

All pregnant women $<14$ weeks of gestation, who received prenatal care from governmental health care systems were eligible for enrollment, except where the following specific exclusion criteria prevented this: Maternal age $<18$ years, preexisting diabetes, date of last menstrual period not certain, no ultrasound estimation from 6 to 14 weeks of gestational age available, chronic hypertension, asthma or currently receiving treatment with oral glucocorticoids, $\beta$-blockers, oral $\beta$-mimetics, Dilantin, or antiretroviral agents and past history of bariatric surgery.

All participants received standard prenatal care recommended by the American College of Obstetricians and Gynecologists (ACOG) [41]. Moreover, participants were scheduled to have two phases of GDM screening in the first and second trimesters of pregnancy, based on the pre-specified protocol for GDM screening, selected for each city.

At each prenatal visit, standardized questionnaires were administered to document prenatal as well as other data needed for research by trained midwives.

\section{Sample size calculation}

Based on previous studies, we assumed that the primary event rate of macrosomia to be equal to $10 \%$ for all groups with no difference. To obtain a statistical power of $85 \%$ with a 1-sided type one error of 0.005 (considering multiple comparisons) approximately 4700 patients per group are needed to show the non-inferiority of more intensive compared to lower intensive strategies with a marginal difference of 0.03 . With a design effect of 0.001 (for cluster sampling) and loss to follow-up of $11 \%$, sample size reached to 5200 in each group [42].

In addition, superiority analyses will be designed to show that one screening strategy is superior to another after non-inferiority has been demonstrated.

\section{Randomization and allocation}

Initially all provinces of Iran were categorized to five stratum based on their geographic location (North, East, West, South, and Center of Iran) and one province in each stratum were randomly selected; then, the list of the cities located in each province were provided. Since the socioeconomic status in the center of provinces may differ from other cities, in the second phase, all cities in each province were classified in two clusters of center of the province and other cities. At the end, four cities were randomly selected from the list of other cities in each province.

For allocation of protocols, in the cluster of the provincial centers, five different protocols were randomly allocated to each provincial center. Also, in the cluster of other cities, four other cities in each province were randomly allocated to the rest of the protocols (Fig. 1). Sample size for each city was estimated through probability proportional to size (PPS), defined by number of live births of the cities.

\section{Intervention}

Following the approval of this study, the study procedure was released as a guideline to all the selected cities. In this respect, workshops were conducted in each city to introduce the study protocol and train the caregivers and study staff accordingly. Dieticians, obstetricians, internal medics, laboratory technicians and endocrinologists in each province were invited to a scientific workshop to harmonize and coordinate the follow ups and treatment of GDM patients. Scientific teams with specialists and executive members conducted visits every 2 months. A telegram channel was developed for daily online communication of scientific members and executive members at both provincial and city levels to answer questions and solve any problems encountered.

Along with routine standard prenatal care, all pregnant women was screened for GDM based on the pre-specified protocol assigned to each city. In this respect, early screening of GDM was conducted in the first trimester of pregnancy, using fasting plasma glucose (FPG) from 


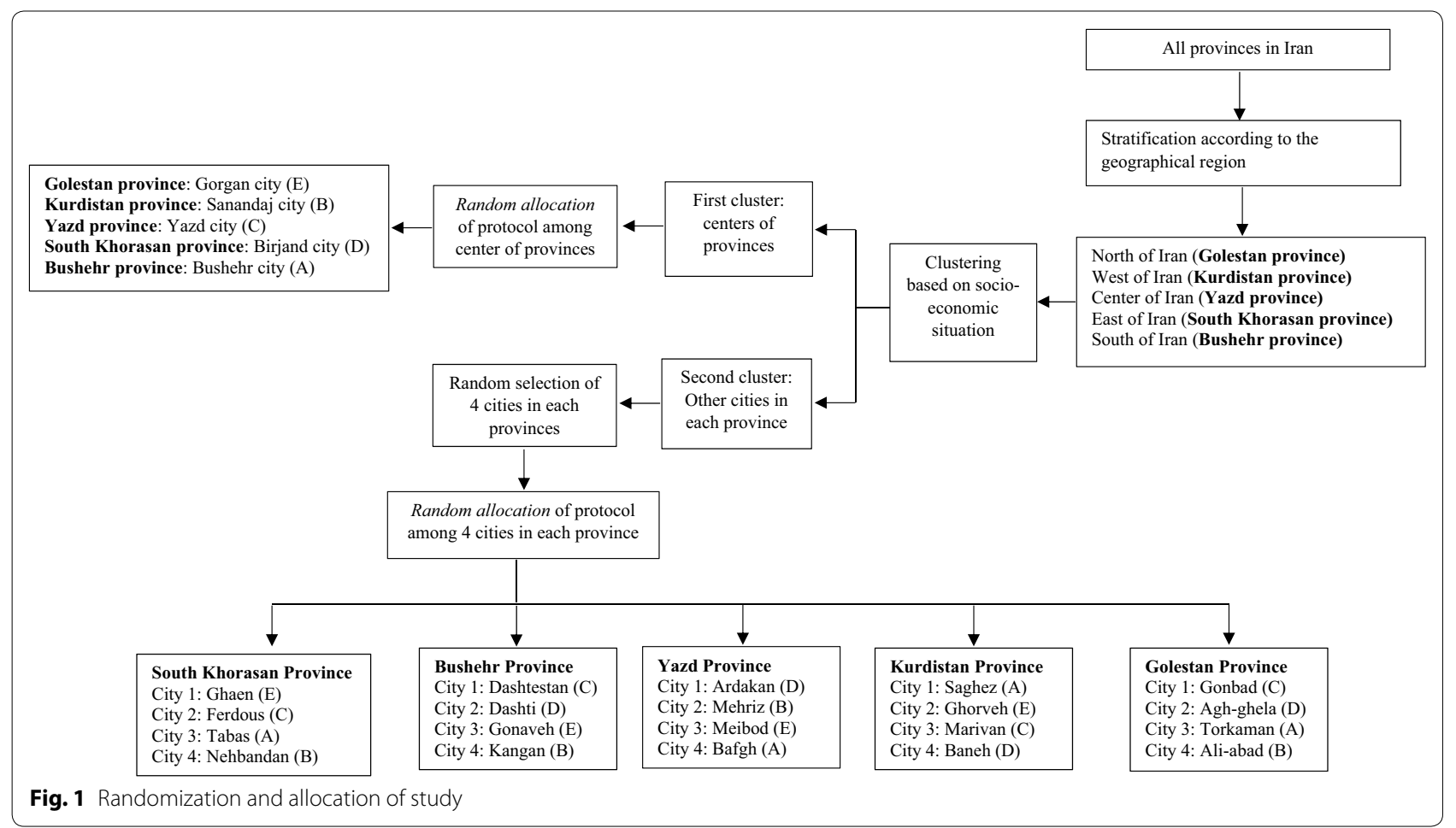

venous sample with the specific threshold based on each screening protocol; based on the results of those screening tests, pregnant women were classified in to three groups: (i) diagnosed with preexisting pre-gestational overt diabetes; (ii) gestational diabetes and (iii) nonGDM women. In addition, at 24-28 weeks of gestation, those not previously known to have diabetes (overt or gestational), were screened again for GDM based on prespecified protocol criteria assigned to that city. All study participants were followed till delivery and pregnancy and neonatal outcomes and health cost were recorded in detail. Definitions of various protocols for screening are presented in Table 1.

Each group received packages of standard care based on their health status. In this respect, non-GDM pregnant women received routine standard care recommended by the American College of Obstetricians and Gynecologists (ACOG) 2013 [41]. Moreover, pregnant diabetic patients received specific prenatal and diabetic care, recommended by the American College of Obstetricians and Gynecologists (ACOG) 2013 [43] and the American Diabetes Association (ADA) 2016 [44].

\section{Summary of management of Gestational Diabetes in Pregnancy}

After diagnosis of GDM, treatment was initiated with medical nutrition therapy, physical activity, and weight management and blood glucose monitoring to achieve the targets recommended by ADA guideline 2016 [44] including fasting, $95 \mathrm{mg} / \mathrm{dL}, 1-\mathrm{h}$ postprandial, $140 \mathrm{mg} /$ $\mathrm{dL}$ or 2 -h postprandial, $120 \mathrm{mg} / \mathrm{dL}$. Medical nutrition therapy for GDM will be individually planed for participants by the dietitian. The food plan provides enough calorie intake to promote fetal/neonatal and maternal health, achieve glycemic goals, and promote appropriate gestational weight gain, based on the Dietary Reference Intakes (DRI) recommendation including a minimum of $175 \mathrm{~g}$ carbohydrate, a minimum of $71 \mathrm{~g}$ protein, and $28 \mathrm{~g}$ fiber [44].

If women did not achieve glycemic goals within 2 weeks, pharmacologic therapy will be offered by specialized physicians including obstetricians, internists or endocrinologists at the second level of the healthcare delivery system. Insulin is the first-line agent recommended for treatment of GDM. Self-monitoring of blood glucose (SMBG) was used for achieving and maintaining therapeutic goals in insulin-treated patients. The frequent use of capillary blood glucose tests of SMBG was scheduled four times a day, fasting, 2-h after breakfast, lunch and dinner or if the patients had hypoglycemic symptoms for at least 2 weeks. After achieving the therapeutic target, SMBG was performed two times a day. In addition, if women decline insulin therapy, metformin will be offered as an alternative or 
Table 1 Definitions of various protocols for screening of gestational diabetes mellitus

\begin{tabular}{|c|c|c|c|c|}
\hline \multirow[t]{2}{*}{ Protocol } & \multirow{2}{*}{$\begin{array}{l}\text { First trimester } \\
\text { Diagnostic criteria for GDM }\end{array}$} & \multicolumn{3}{|l|}{ Second trimester } \\
\hline & & Method for GDM screening & Diagnostic threshold of test & Diagnostic criteria \\
\hline A & $92 \mathrm{mg} / \mathrm{dL}<\mathrm{FPG}>126 \mathrm{mg} / \mathrm{dL}$ & One step with 2-h $75 \mathrm{~g}$ OGTT & $\begin{array}{l}\text { Fasting } \geq 92 \mathrm{mg} / \mathrm{dL} \\
1 \mathrm{~h} \geq 180 \mathrm{mg} / \mathrm{dL} \\
2 \mathrm{~h} \geq 153 \mathrm{mg} / \mathrm{dL}\end{array}$ & $\begin{array}{l}\text { GDM is defined as any of the given } \\
\text { plasma glucose values are met or } \\
\text { exceeded }\end{array}$ \\
\hline B & $100 \mathrm{mg} / \mathrm{dL}<\mathrm{FPG}>126 \mathrm{mg} / \mathrm{dL}$ & One step with 2-h 75 g OGTT & $\begin{array}{l}\text { Fasting } \geq 92 \mathrm{mg} / \mathrm{dL} \\
1 \mathrm{~h} \geq 180 \mathrm{mg} / \mathrm{dL} \\
2 \mathrm{~h} \geq 153 \mathrm{mg} / \mathrm{dL}\end{array}$ & $\begin{array}{l}\text { GDM is defined as two or more of the } \\
\text { given plasma glucose values are } \\
\text { met or exceeded }\end{array}$ \\
\hline C & $100 \mathrm{mg} / \mathrm{dL}<\mathrm{FPG}>126 \mathrm{mg} / \mathrm{dL}$ & One step with 2-h $75 \mathrm{~g}$ OGTT & $\begin{array}{l}\text { Fasting } \geq 92 \mathrm{mg} / \mathrm{dL} \\
1 \mathrm{~h} \geq 180 \mathrm{mg} / \mathrm{dL} \\
2 \mathrm{~h} \geq 153 \mathrm{mg} / \mathrm{dL}\end{array}$ & $\begin{array}{l}\text { GDM is defined as any of the given } \\
\text { plasma glucose values are met or } \\
\text { exceeded }\end{array}$ \\
\hline $\mathrm{D}$ & $92 \mathrm{mg} / \mathrm{dL}<\mathrm{FPG}>126 \mathrm{mg} / \mathrm{dL}$ & $\begin{array}{l}\text { Two steps with } 50 \mathrm{~g} \mathrm{GCT}-1 \mathrm{~h} \\
\text { following } \\
\text { 3-h } 100 \mathrm{~g} \text { OGTT }\end{array}$ & $\begin{array}{l}50 \mathrm{~g} \mathrm{GCT}: \\
\text { BS-1 } \mathrm{h}: \geq 140 \mathrm{mg} \\
100 \mathrm{~g} \text { OGTT: } \\
\text { Fasting } \geq 95 \mathrm{mg} / \mathrm{dL} \\
1 \mathrm{~h} \geq 180 \mathrm{mg} / \mathrm{dL} \\
2 \mathrm{~h} \geq 155 \mathrm{mg} / \mathrm{dL} \\
3 \mathrm{~h} \geq 140 \mathrm{mg} / \mathrm{dL}\end{array}$ & $\begin{array}{l}\text { GDM is defined as if two or more of } \\
\text { the given plasma glucose values in } \\
100 \mathrm{~g} \text { OGTT are met or exceeded }\end{array}$ \\
\hline $\mathrm{E}$ & $100 \mathrm{mg} / \mathrm{dL}<\mathrm{FPG}>126 \mathrm{mg} / \mathrm{dL}$ & $\begin{array}{l}\text { Two steps with } 50 \mathrm{~g} \mathrm{GCT}-1 \mathrm{~h} \\
\text { following } \\
\text { 3-h } 100 \mathrm{~g} \text { OGTT }\end{array}$ & $\begin{array}{l}50 \mathrm{~g} \mathrm{GCT}: \\
\text { BS-1 } \mathrm{h}: \geq 140 \mathrm{mg} \\
100 \mathrm{~g} \text { OGTT: } \\
\text { Fasting } \geq 95 \mathrm{mg} / \mathrm{dL} \\
1 \mathrm{~h} \geq 180 \mathrm{mg} / \mathrm{dL} \\
2 \mathrm{~h} \geq 155 \mathrm{mg} / \mathrm{dL} \\
3 \mathrm{~h} \geq 140 \mathrm{mg} / \mathrm{dL}\end{array}$ & $\begin{array}{l}\text { GDM is defined as if two or more of } \\
\text { the given plasma glucose values in } \\
100 \mathrm{~g} \mathrm{OGTT} \text { are met or exceeded }\end{array}$ \\
\hline
\end{tabular}

In the first trimester overt diabetes is defined as FPG $\geq 126 \mathrm{mg} / \mathrm{dL}$

FPG fasting plasma glucose, GCT glucose challenge test, OGTT oral glucose tolerance test

adjunct to insulin after clarifying the harms and benefits of metformin therapy for patients [44] (Fig. 2).

\section{Data collection}

Data were collected from participants at scheduled time points (Table 2) using pre-specified questionnaires and clinical and para clinical exams by trained midwives. Moreover, data on neonatal mortalities that occurred after hospital discharge were collected at 4 weeks postpartum by telephone and subsequent reviews of medical records.

\section{Questionnaires}

1. Prenatal questionnaire This comprehensive questionnaire includes two sections: 1-contains the past medical, reproductive, obstetrics, and gynecological history, completed only at first prenatal visit 2-focuses on current pregnancy information and this part was completed at each prenatal visit during pregnancy (Additional file 1: PART 1: Prenatal Care Form).
2. Delivery, postpartum and neonatal questionnaire This questionnaire contains the details of delivery and its methods and any adverse maternal-fetal/neonatal outcomes (Additional file 1: PART 2. Childbirth and New-born Report Form).

3. Quality of life questionnaire The Iranian version of 36-item short form health survey questionnaire (SF36) [45-48] was used to measure the physical and mental components of health-related quality of life. The SF-36 included 36 items with 8 subscales; physical functioning, role limitations due to physical problems, bodily pain, general health perceptions, vitality, social functioning, role limitations due to emotional problems and perceived mental health. This questionnaire was completed monthly for all GDM patients since the time of diagnosis. Also, it were done for $5 \%$ of non-GDM pregnant women visited from the first visit for prenatal care (Additional file 1: PART 3. 36-Item Short Form Survey Instrument).

4. Cost-effectiveness questionnaire This questionnaire included 50 items with three subscales: (i) self-purchased health care, (ii) travel costs for making return visit(s) to health care and (iii) time costs of travel- 


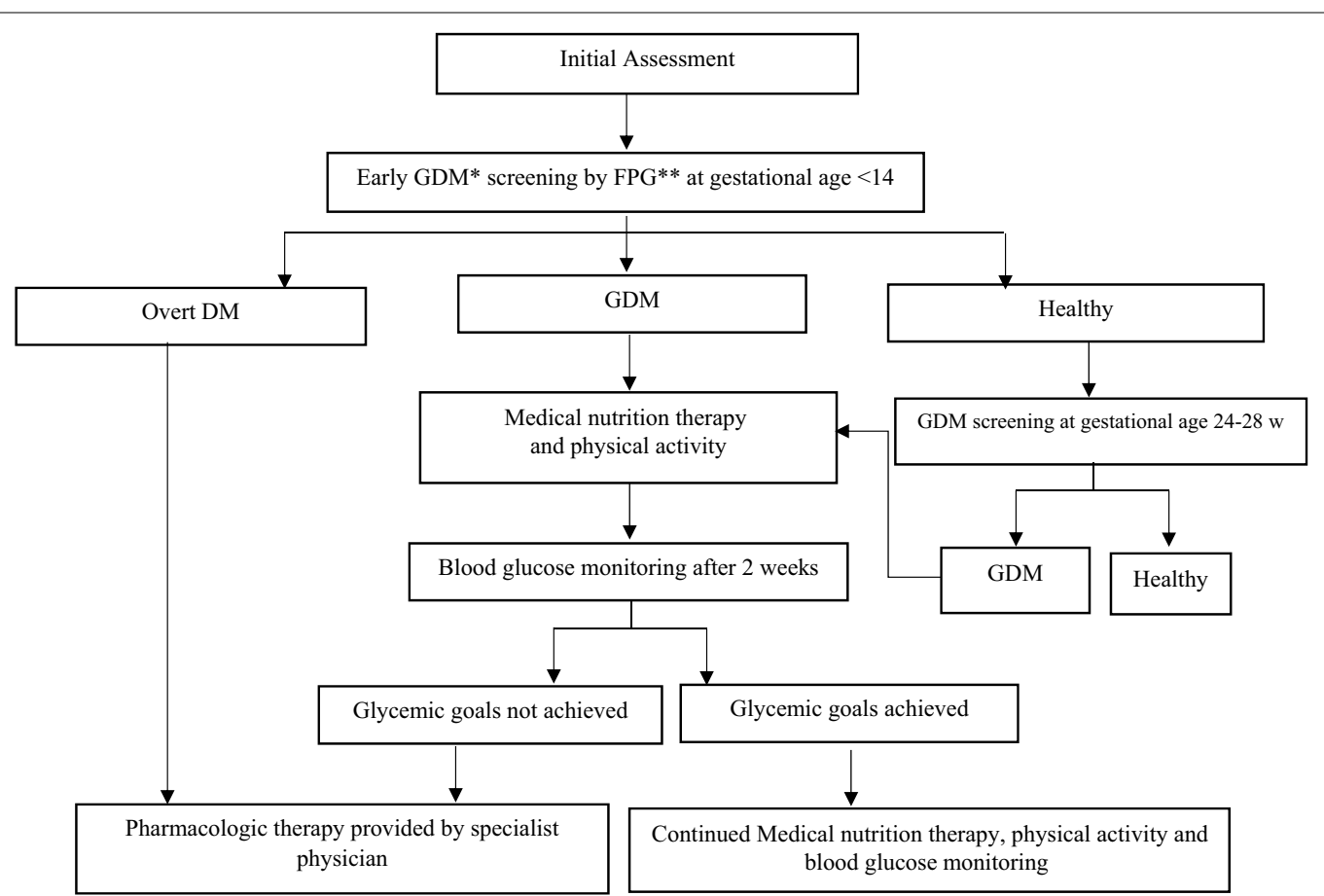

Fig. 2 Flow chart of screening and management of Gestational Diabetes in Pregnancy. *GDM: gestational diabetes mellitus; **FPG: fasting plasma glucose

ling and attending health care center. Effectiveness was measured in terms of quality adjusted life years (QALYs), using the EQ-5D 3L questionnaire completed by participants at the follow up time points. It includes five questions, each assessing one of five dimensions of the health related quality of life (Mobility, Self-Care, Usual Activities, Pain/Discomfort and Anxiety/Depression). Each of these dimensions has to be answered on a 3-level scale (no problems, some or moderate problems, and extreme problems). The scales are scored from 1 (no problem) to 3 (extreme problem) in each question; and finally the score digits are placed together to yield a 5-digit code for the health status of each patient (Additional file 1: PART 4. Cost effectiveness Form).

\section{Maternal anthropometric, clinical, and laboratory assessments}

Weight was measured to the nearest $100 \mathrm{~g}$ using digital scales while the participants were minimally clothed, without shoes. Height was measured to the nearest $0.5 \mathrm{~cm}$, in a standing position without shoes, using a tape measure, while shoulders were in normal alignment. Body mass index (BMI) was calculated as weight $(\mathrm{kg})$ divided by height squared $\left(\mathrm{m}^{2}\right)$. After a 15-min rest in the sitting position, two measurements of systolic and diastolic blood pressure (SBP and DBP) were taken on the right arm, using a standardized mercury sphygmomanometer (calibrated by the Iranian Institute of Standards and Industrial Researches); the mean of the two measurements was considered as the participant's blood pressure.

Plasma glucose were measured on the day of blood collection. A blood sample was drawn between 7:00 and 9:00 AM from all study participants, after 8 to $10 \mathrm{~h}$ overnight fasting. For the 75-g OGTT-82.5 g of glucose monohydrate solution (equivalent to $75 \mathrm{~g}$ anhydrous glucose), for the $50 \mathrm{~g}$ glucose challenge test (GCT)-55 g of glucose monohydrate solution (equivalent to $50 \mathrm{~g}$ anhydrous glucose) and for the 100-g OGTT-100 g of glucose monohydrate solution (equivalent to $110 \mathrm{~g}$ anhydrous glucose) were administered orally to subjects and plasma glucose was measured, using an enzymatic colorimetric method with glucose oxidase; inter- and intra-assay coefficients of variation were less than $2.3 \%$. Analyses were performed using Pars Azmon kits (Pars Azmon Inc., Tehran, Iran) using the Selectra 2 auto-analyzer (Vital Scientific, Spankeren, Netherlands).

\section{Neonatal anthropometric, clinical, and laboratory assessments}

Neonatal anthropometric and clinical measurement were measured by trained staff. Birth weight was measured without diapers using a calibrated digital baby scale 


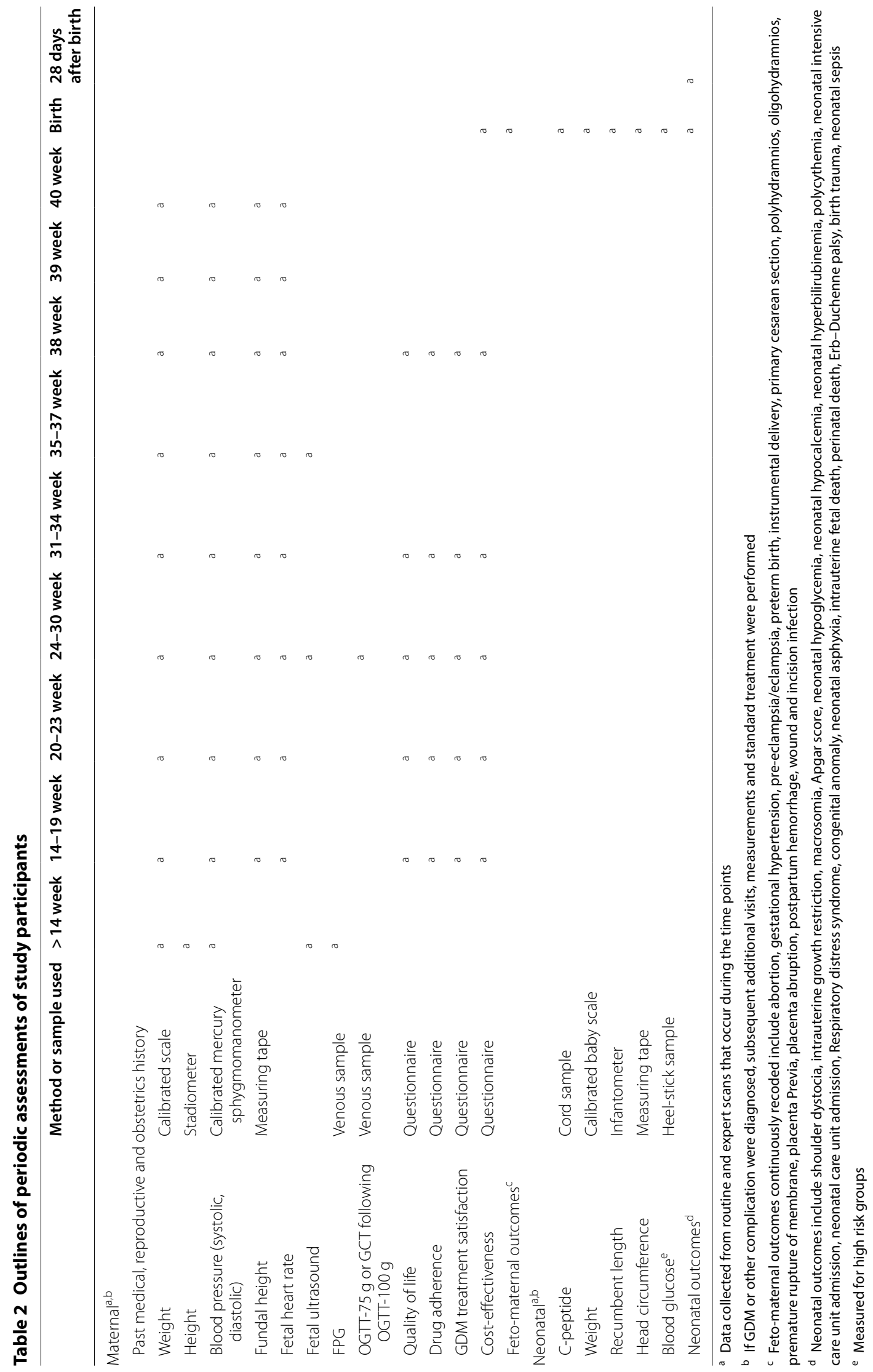


(SECA model 334; SECA Corp., Hamburg, Germany) to the nearest $1 \mathrm{gr}$, within an hour after delivery. Recumbent length was measured to nearest $0.1 \mathrm{~cm}$ from the top of the head to the sole of the feet using an infantometer (Easy-Glide Bearing Infantometer, Perspective Enterprises). Head circumference ( $\mathrm{HC}$ ) was measured at the largest occipito-frontal diameter and the measurement was rounded to the nearest $0.25 \mathrm{~cm}$. The largest of three consecutive measurements was recorded.

In this respect, two measurements were obtained, and if results differed by $>10 \mathrm{~g}$ for weight and $0.5 \mathrm{~cm}$ for length or head circumference, a third measurement were taken. The average of the two or three measurements was used for final analysis.

According to the national Iranian guidelines, all newborns were exclusively breastfed early after delivery. Infants were either screened for hypoglycemia $1-2 \mathrm{~h}$ after birth before a feeding based on the presence of defined risk factors including maternal GDM/overt DM, birth weight $>90$ th percentile, maternal $\mathrm{BMI}>30$, birth weight $<10$ th percentile, early preterm birth less than 34 weeks of gestation, perinatal acidosis, 5-min Apgar score of $0-3$, failure of breastfeed and sepsis.

In this respect, blood glucose levels were measured using heel-stick sampling at $1,3,6,12$, and $24 \mathrm{~h}$ after birth before a feeding. Additional blood glucose measurements were performed in case of hypoglycemia or clinical symptoms including sweating, weak or high-pitched cry, feeding difficulties, poor sucking, tremors, hypothermia, irritability, lethargy/stupor, hypotonia, seizures, apnea, grunting or tachypnea or cyanosis. Using point-of-care testing, glucose was measured with the glucose oxidase method (Pars Azmon Inc., Tehran, Iran).

Cord serum C-peptide sample, as the index of fetal $\beta$-cell function, was collected at the time of delivery in a subsample of 1000 participants with different screening protocol. Samples collected were centrifuged for $10 \mathrm{~min}$ at $3000 \mathrm{rpm}$, stored at $-80{ }^{\circ} \mathrm{C}$ and transferred to central laboratory. C-peptide were determined with ELISA method (Mercodia AB, Uppsala, Sweden); the inter- and intra-assay coefficient of variation were $<2.3 \%$ and $1.5 \%$, respectively.

The need for other assessments, such as serum bilirubin or imaging tests were determined based on clinical indications.

\section{Definition of study outcomes}

Outcomes of study were defined as follows: Macrosomia/large for gestational age (LGA) was defined as birth-weight $>4000 \mathrm{~g}$ and/or fetal-weight $>90$ th percentile for a given gestational age [49] using ultrasound biometry for estimating the fetal-weight and multinational World Health Organization (WHO) fetal growth chart for defining the percentile. Primary cesarean section was defined as the cesarean deliveries out of all births to women who had not had a previous cesarean delivery [50]; abortion refers to a termination of a pregnancy either natural or induced before the completion of 20 weeks of gestation. Polyhydramnios is defined as excess accumulation of amniotic fluid with 4-quadrant amniotic fluid index (AFI) more than $24 \mathrm{~cm}$ or a single maximum vertical pocket more than $8 \mathrm{~cm}$ [51]. Oligohydramnios refers to decreased amniotic fluid volume relative to gestational age with AFI less than $24 \mathrm{~cm}$ or a single maximum vertical pocket less than $8 \mathrm{~cm}$ [52]. Intrauterine growth restriction (IUGR)/fetal growth restriction was defined as fetal-weight less than the 10th percentile for gestational age [53] using ultrasound biometry for estimating the fetal-weight and multinational World Health Organization (WHO) fetal growth chart for defining the percentile. Small size for gestational age (SGA) refers to birth-weight less than the 10th percentile for gestational age $[53,54]$ using gender specific WHO weight-for-age chart for defining the percentile. Hypoglycemia was defined as plasma glucose concentration $<47 \mathrm{mg} / \mathrm{dL}$ in the first $48 \mathrm{~h}$ after delivery $[55,56]$; hyperbilirubinemia was determined by value greater than the 95th percentile for any given point after birth [57]; Gestational hypertension was defined as a systolic pressure of $\geq 140 \mathrm{mmHg}$ or a diastolic pressure of $\geq 90 \mathrm{mmHg}$ taken on two occasions, at least $4 \mathrm{~h}$ apart [58, 59]; Preeclampsia was defined as an elevation in blood pressure $\geq 140 \mathrm{mmHg}$ systolic or $\geq 90 \mathrm{mmHg}$ diastolic on two occasions at least $4 \mathrm{~h}$ apart after 20 weeks of gestation in a women with a previously normal blood pressure and proteinuria $\geq 300 \mathrm{mg}$ per $24 \mathrm{~h}$ urine collection or protein/creatinine ratio greater than or equal to 0.3 or dipstick reading of $1+$ and more if other quantitative methods were not available. In the absence of proteinuria, new-onset hypertension with the new onset of any of the thrombocytopenia, renal insufficiency, impaired liver function, pulmonary edema and cerebral or visual symptoms [59]; preterm birth was defined as when birth occurs between 20 and 37 weeks of pregnancy [60]; shoulder dystocia was defined clinically, where providers are required to provide additional obstetric maneuvers when gentle downward traction has failed to affect the delivery of the shoulders [61] and birth trauma was defined as brachial plexus palsy or clavicular, humeral, or skull fracture. Mild GDM is defined as: a fasting glucose level of $>92$ and $<100 \mathrm{mg}$ per decilitre in 1st trimester of pregnancy and only one glucose measurement exceeding from established thresholds for 2-h 75gOGTT as follows: FPG $>92 \mathrm{mg} / \mathrm{dL}, 1$-h plasma glucose $>180 \mathrm{mg} / \mathrm{dL}$, 2-h plasma glucose $>53 \mathrm{mg} / \mathrm{dL}$ at the 24-28 weeks of gestation. 


\section{Data cleaning and missing data}

The following minimal data must be available for women to be included in the analysis of pregnancy outcomes: Completed enrollment forms and questionnaire, completed results of GDM screening, type of delivery, birth weights and clear status of exclusionary criteria.

Missing values will be managed using appropriate imputation methods. Outliers will be identified using graphical tools including boxplot and/or Model-based methods like Chauvenet's criterion and Dixon's Q test $[62,63]$.

\section{Data analysis}

To illustrate distribution of the data, appropriate descriptive statistics such as measures of central tendency, index of dispersion and percentiles will be reported along with normality assumption testing through KolmogorovSmirnoff test. Maternal, neonatal and obstetric outcomes of the 4 less intensive screening strategies with IADPSG criteria will be compared using parametric or non-parametric statistical tests, where applicable.

In addition, based on the type of outcome variables, Generalized Linear Models (GLMs) with different link function such as linear, count or binary will be applied. Stepwise method with P-value $<0.2$ will be used to identify significant confounding variables and estimate adjusted measures of interests. Moreover, longitudinal modeling through Generalized Estimating Equation (GEE) analysis approach will be conducted and to calculate Number Needed to Treat (NNT), the Linear GLM model will be applied as well. Since this is a cluster randomized trial, cluster effect in analysis will be considered.

\section{Cost-effectiveness analysis (CEA)}

A cost-effectiveness analysis, comparing 4 less intensive screening strategies with IADPSG criteria will be conducted on an intention-to-treat basis by estimating various parameters including Quality-adjusted life years (QALYs), incremental cost-effectiveness ratio (ICER), and incremental net benefit (INB). To estimate mean cost in each treatment group, regression models will be used. General linear models (GLM) with appropriate variance functions e.g. gamma, Poisson, etc. and link will be used to identify the relationship between treatment allocation and costs after adjusting for minimization and the appropriate prognostic covariates at baseline (e.g. Baseline EQ-5D score). To estimate the incremental effect of the treatment indicator variable, recycled predictions will be used [64].

A sensitivity analysis will be conducted to assess how sensitive the cost-effectiveness results are to variation in key parameters including cost.

\section{Bayesian and Markov Modeling}

Bayesian Cost Effectiveness Modeling (BCEM) will be used to overcome the complexity of the relationships linking a suitable measure of clinical benefit (e.g. qualityadjusted life years) and the associated costs. Simplifying assumptions, such as normality of the underlying distributions, are usually not granted, particularly for the cost variable, which is significantly skewed distributions. In addition, individual-level data sets are often characterized by the presence of structural zeros in the cost variable [65-67]. Bayesian models will be used to account for the presence of excess zeros in a distribution and have been applied in the context of cost data (Fig. 3).

Markov model will be used to extrapolate the results of the trial beyond the follow up, which will eventually provide longer-term cost-effectiveness. Markov decision processes (MDPs) are a powerful and appropriate technique for modelling medical decision. MDPs are most useful in classes of problems involving complex, stochastic and dynamic decisions like medical treatment decisions, for which they can find optimal solutions [68]. Physicians will always need to make subjective judgments about treatment strategies, but mathematical decision models can provide insight into the nature of optimal choices and guide treatment decisions [69]. Markov models can be used to describe various health states in a population of interest, and to detect the effects of various policies or therapeutic choices. In addition, we will apply decision tree analysis and then apply probabilistic approach.

All data analysis will be conducted using $\mathrm{R}$ (Version 2.2.2) and TreeAge (Version 13) softwares.

\section{Approval and ethical considerations}

This trial has been approved and funded by the National Institute for Medical Research Development under Grant Agreement No IR.NIMAD.REC.1394.013. Funding source had no involvement in the study. The protocol was approved by the national ethics committee of the National Institute for Medical Research Development (Approval number: IR.NIMAD.REC.1394.013). In addition, the Iranian Ministry of Health and Medical Education (MoHME) approved the study protocol and pre specified GDM modalities were made available to all those provinces as mandatory guidelines. This field trial has been registered in Iranian Registry of Clinical Trials (Trial Registration: IRCT138707081281N1).

\section{Discussion}

At present, there is a lack of international consensus about the diagnosis of gestational diabetes. Screening strategies, testing methods and even diagnostic optimum glycemic thresholds for GDM remain the subject 


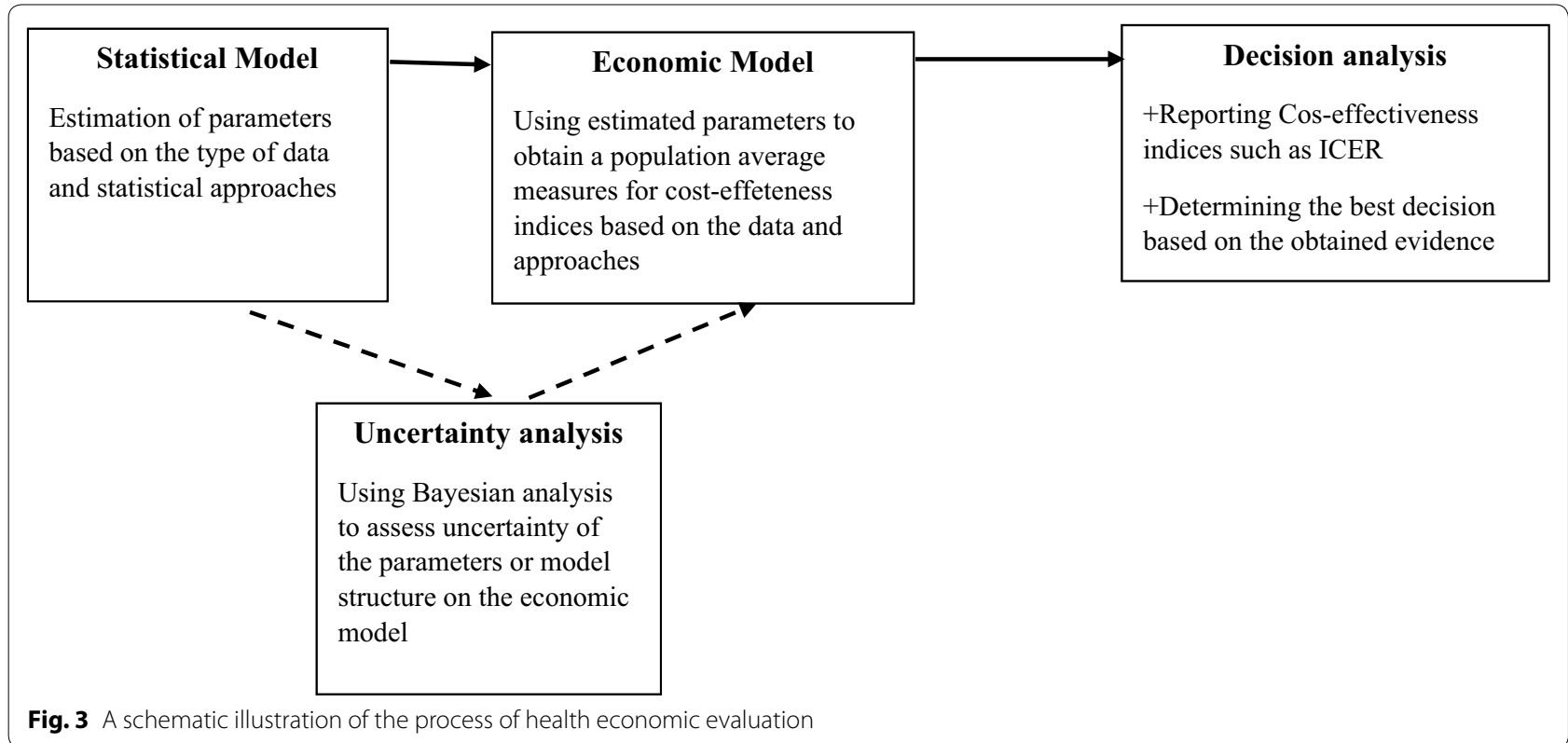

of considerable debate. Although gestational diabetes mellitus is a recognized marker for an increased risk of subsequent diabetes, its clinical significance with respect to its various definitions and various adverse pregnancy outcomes has not been clearly elucidated. Women with severe gestational diabetes and highly elevated fasting plasma glucose levels apparently are at an increased risk for adverse pregnancy outcomes if treatment is not provided, yet the association of milder forms of gestational diabetes with such outcomes remains unclear. Despite the HAPO study having provided valuable evidence of the association of maternal blood glucose with adverse pregnancy outcomes, it is worth noting that HAPO study was a purely observational study that conducted in western countries.

Considering the fact that majority of births annually occur in low- and low-middle income countries with high prevalence of GDM and limited resources [5], the cost-effectivity of this definition needs to be re-evaluated in other communities; the present study will hopefully provide such information from an eastern Mediterranean region. Moreover there is little information comparing the clinical efficacy, utility and feasibility of the two step GDM screening test and a 3-h oral glucose tolerance test (GTT) and the one step oral glucose tolerance test (OGTT) approaches, our study will provide comprehensive data on this comparison in the same population.

According to a WHO report, global and local decision making regarding GDM strategies are challenging due to the lack of optimum economic evaluations of various GDM screening protocols; as a result our study will provide the data needed for each community to adopt its specific GDM screening guidelines according to the reasonable cost for prevention of the adverse short and long term effects of GDM.

The limitations of our study of course should be addressed. Since specific questionnaires for evaluation of QOL and drug adherence in patients with GDM were not available, general questionnaires was used. In addition, we did not use the central reference laboratory for all of our measurement except C-peptide. Since homogeneity of laboratory procedures are essential to the success the study, we used standardized procedures in all provinces including local training of field center laboratory personnel, using a common protocol for measurement of glucose; using of standard equipment and supplies; monthly external quality controls for each laboratory. Moreover, glycosylated hemoglobin A1c (HbA1c) measurements were not available in our study.

\section{Conclusions}

Results which if needed, will also enable policy makers to optimize the national GMD strategy as a resource for enhancing GDM guidelines.

\section{Supplementary information}

Supplementary information accompanies this paper at https://doi. org/10.1186/s13098-019-0493-z.

Additional file 1: PART 1: Prenatal Care Form; PART 2. Childbirth and New-born Report Form; PART 3. 36-Item Short Form Survey Instrument; PART 4. Cost effectiveness Form. 


\section{Abbreviations}

GDM: gestational diabetes mellitus; IADPSG: the International Association of Diabetes in Pregnancy Study Group; OGTT: oral glucose tolerance test: WHO: World Health Organization; ACOG: American College of Obstetricians and Gynecologists; FPG: fasting plasma glucose; ADA: American Diabetes Association; DRI: Dietary Reference Intakes; QALYs: quality adjusted life years; AFI: amniotic fluid index; SGA: small size for gestational age; IUGR: intrauterine growth restriction; GLMs: Generalized Linear Models; GEE: Generalized Estimating Equation; INB: incremental net benefit; ICER: incremental cost-effectiveness ratio; BCEM: Bayesian Cost Effectiveness Modeling.

\section{Acknowledgements}

We thank from Golestan, Bushehr, Birjand, Kurdistan and Yazd Universities of medical Sciences for their cooperation for this study.

\section{Gulf Study Cooperative Research Group}

Samira Behboudi-Gandevani, PhD (Reproductive Endocrinology Research Center, Research Institute for Endocrine Sciences, Shahid Beheshti University of Medical Sciences, Tehran, Iran);

Mehrandokht Abedini, MD (Maternal health Department, Ministry of Health and Medical Education, Tehran, Iran);

Masoud Soleymani-Dodaran, MD, PhD, MPH, FFPH, CCT (Minimally Invasive Surgery Research Center, Iran University of Medical Sciences, Tehran, Iran);

Davood Khalili, MD, MPH, PhD (Prevention of Metabolic Disorders Research Center, Research Institute for Endocrine Sciences, Shahid Beheshti University of Medical Sciences, Tehran, Iran);

Farshad Farzadfar, MD (Non-Communicable Diseases Research Center, Endocrinology and Metabolism Research Institute, Tehran University of Medical Sciences, Tehran, Iran);

Farhad Hoseinpanah, MD (Prevention of Metabolic Disorders Research Center, Research Institute for Endocrine Sciences, Shahid Beheshti University of Medical Sciences, Tehran, Iran);

Farzad Hadaegh, MD (Prevention of Metabolic Disorders Research Center, Research Institute for Endocrine Sciences, Shahid Beheshti University of Medical Sciences, Tehran, Iran);

Majid Valizadeh, MD (Obesity Research Center, Research Institute for Endocrine Sciences, Shahid Beheshti University of Medical Sciences, Tehran, Iran);

Farahnaz Torkestani, MD (Department of Obstetrics and Gynecology, Faculty of Medicine, Shahed University of Medical Sciences, Tehran, Iran);

Zahra Abdollahi, PhD (Nutrition Department, Undersecretary of Public Health, Ministry of Health \& Medical Education, Tehran, Iran);

Marzieh Bakhshandeh, MSc (Maternal health Department, Ministry of Health and Medical Education, Tehran, Iran);

Razieh Bidhendi Yarandi, PhD (Department of Epidemiology and Biostatistics, School of Public Health, Tehran University of Medical Sciences, Tehran, Iran);

Mehdi Zokaee, MD (Population, family and school health Department, Kurdistan University of Medical Sciences, Sanandaj, Iran);

Farzam Bidarpour, MD (Deputy of Health, Kurdistan University of Medical Sciences, Sanandaj, Iran);

Mehdi Javanbakht, PhD (Health Economics Research Unit, Institute of Applied Health Sciences, University of Aberdeen, UK);

Iraj Nabipour, MD (The Persian Gulf Biomedical Sciences Research Institute, Bushehr University of Medical Sciences);

Mohammad Ali Mansournia, MD (Department of Epidemiology and Biostatistics, School of Public Health, Tehran University of Medical Sciences, Tehran, Iran):

Ensieh Nasli Esfahani, MD (Diabetes Research Center, Endocrinology and Metabolism Research Institute, Tehran University of Medical Sciences, Tehran, Iran);

Afshin Ostovar, MD (The Persian Gulf Biomedical Sciences Research Institute, Bushehr University of Medical Sciences, Bushehr, Iran);

Fereidoun Azizi, MD (Endocrine Research Center, Research Institute for Endocrine Sciences, Shahid Beheshti University of Medical Sciences, Tehran, Iran) and Executive committee.

\section{Gulf Study Cooperative Executive committee}

Abbas Najari, MD (Centre for Collective Reflection \& Implementation of Ideas, Undersecretary for Research and Technology, Ministry of Health and Medical Education, Tehran, Iran);
Abdolmohhamad Khajeian, MD (Deputy of Health, Bushehr University of Medical Sciences, Bushehr, Iran);

Azita Anaraki, MD (Population, family and school health Department,

Bushehr University of Medical Sciences, Bushehr, Iran);

Fariba Ghazaghi, MSc (Population, family and school health Department, Birjand University of Medical Sciences, Birjand, Iran);

Forouzan Lahouni, MS (Population, family and school health Group, Kurdistan University of Medical Sciences, Sanandaj, Iran);

Forouzandeh Kalantari, MD (Population, family and school health Department, Yazd University of Medical Sciences, Yazd, Iran);

Hossein Fallah, MSc (Nutrition Department, Ministry of Health and Medical

Education, Tehran, Iran);

Khadije Kordi, MD (Population, family and school health Department,

Golestan University of Medical Sciences, Gorgan, Iran);

Lotfollah Saed, MD (Department of Internal Medicine, Faculty of Medicine, Kurdistan University of Medical Sciences, Sanandaj, Iran);

Mahsa Norooozzadeh, MSc (Reproductive Endocrinology Research Center, Research Institute for Endocrine Sciences, Shahid Beheshti University of Medical Sciences, Tehran, Iran);

Maryam Farahmand, PhD (Reproductive Endocrinology Research Center, Research Institute for Endocrine Sciences, Shahid Beheshti University of Medical Sciences, Tehran, Iran);

Marzieh Rostami Dovom, PhD (Reproductive Endocrinology Research Center, Research Institute for Endocrine Sciences, Shahid Beheshti University of Medical Sciences, Tehran, Iran);

Mehdi Hedayati, PhD (Cellular and Molecular Research Center, Research Institute for Endocrine Sciences, Shahid Beheshti University of Medical Sciences, Tehran, Iran);

Mehdi Mehdizade, MD (Deputy of Health, Birjand university of Medical science, Birjand, Iran);

Mina Amiri, PhD (Reproductive Endocrinology Research Center, Research Institute for Endocrine Sciences, Shahid Beheshti University of Medical Sciences, Tehran, Iran);

Mohammad Hassan Lotfi, MD (Deputy of health, Kurdistan University of Medical Sciences, Sanandaj, Iran);

Mohammad-Esmaeil Motlagh, MD (Department of Pediatrics, Ahvaz Jundishapur University of Medical Sciences, Ahvaz, Iran);

Mozhgan Bashghareh, MSc (Population, family and school health Depart-

ment, Golestan University of Medical Sciences, Gorgan, Iran);

Nosrat Zamanipour, MSc (Population, family and school health Department, Birjand University of Medical Sciences, Birjand, Iran);

Parvin Mirmiran, PhD (Nutrition and Endocrine Research Center, Research Institute for Endocrine Sciences, Shahid Beheshti University of Medical Sciences, Tehran, Iran);

Saeid Sadeghian Sharif, PhD (Faculty of nutrition Science and Food Technology, Shahid Beheshti University of Medical Sciences, Tehran Iran);

Saeid Shahraz, PhD (Heller School of Social Policy and Management, Brandeis University, Waltham, Massachusetts, USA);

Samareh Khari, MD (Population, family and school health Department,

Golestan University of Medical Sciences, Gorgan, Iran);

Sedigheh Alishahi, MSc (Population, family and school health Department, Yazd University of Medical Sciences, Yazd, Iran);

Shole Shahgheibi, MD (Department of Obstetrics and Gynecology, Faculty of Medicine, Kurdistan University of Medical Sciences, Sanandaj, Iran);

Sima Nazarpour, PhD (Reproductive Endocrinology Research Center, Research Institute for Endocrine Sciences, Shahid Beheshti University of Medical Sciences, Tehran, Iran);

Yadollah Mehrabi, PhD (Department of Epidemiology, School of Health, Shahid Beheshti University of Medical Sciences, Tehran, Iran);

Zahra Ghaedmohammadi, MSc (Population, family and school health Department, Bushehr University of Medical Sciences, Bushehr, Iran).

\section{Authors' contributions}

FRT, SBG, MA, MSD, DKH, FF, FH, FH, MV, FT, ZA, MB, RBY, MZ, FB, IN, SSH, MAM, $E N E, A O, F A$ contributed to conception of the article and study design. All authors contribute for study design in provinces manuscript drafting and critical discussion. All authors read and approved the final manuscript. 


\section{Funding}

This trial has been approved and funded by the National Institute for Medical Research Development under Grant Agreement No IR.NIMAD.REC.1394.013. Funding source had no involvement in the study.

\section{Availability of data and materials}

The datasets used and analyzed during the current study are available from the corresponding author on reasonable request.

\section{Ethics approval and consent to participate}

The protocol was approved by the national ethics committee of the National Institute for Medical Research Development (Approval number: IR.NIMAD. REC.1394.013). In addition, the Iranian Ministry of Health and Medical Education (MoHME) approved the study protocol and pre specified GDM modalities were made available to all those provinces as mandatory guidelines. This field trial has been registered in Iranian Registry of Clinical Trials (Trial Registration: IRCT138707081281N1)

\section{Consent for publication}

Not applicable.

\section{Competing interests}

The authors declare that they have no competing interests.

Received: 19 August 2019 Accepted: 9 November 2019

Published online: 18 December 2019

\section{References}

1. Wang C, Yang HX. Diagnosis, prevention and management of gestational diabetes mellitus. Chronic Dis Transl Med. 2016;2:199-203.

2. Eades CE, Cameron DM, Evans JM. Prevalence of gestational diabetes mellitus in Europe: a meta-analysis. Diabetes Res Clin Pract. 2017:129:173-81.

3. Lee KW, Ching SM, Ramachandran V, Yee A, Hoo FK, Chia YC, et al. Prevalence and risk factors of gestational diabetes mellitus in Asia: a systematic review and meta-analysis. BMC Pregnancy Childbirth. 2018;18:494.

4. Jiwani A, Marseille E, Lohse N, Damm P, Hod M, Kahn JG. Gestational diabetes mellitus: results from a survey of country prevalence and practices. J Matern Fetal Neonatal Med. 2012;25:600-10.

5. Behboudi-Gandevani S, Amiri M, Yarandi RB, Tehrani FR. The impact of diagnostic criteria for gestational diabetes on its prevalence: a systematic review and meta-analysis. Diabetol Metab Syndr. 2019:11:11.

6. Smith KB, Smith MS. Obesity statistics. Prim Care. 2016;43:121-35.

7. Prutsky GJ, Domecq JP, Sundaresh V, Elraiyah T, Nabhan M, Prokop LJ, et al. Screening for gestational diabetes: a systematic review and meta-analysis. J Clin Endocrinol Metab. 2013:98:4311-8.

8. Minooee S, Tehrani FR, Rahmati M, Mansournia MA, Azizi F. Dyslipidemia incidence and the trend of lipid parameters changes in women with history of gestational diabetes: a 15-year follow-up study. Endocrine. 2017;58:228-35

9. Lauenborg J, Mathiesen E, Hansen T, Glumer C, Jørgensen T, BorchJohnsen K, et al. The prevalence of the metabolic syndrome in a Danish population of women with previous gestational diabetes mellitus is three-fold higher than in the general population. J Clin Endocrinol Metab. 2005:90:4004-10

10. Garcia-Vargas L, Addison SS, Nistala R, Kurukulasuriya D, Sowers JR. Gestational diabetes and the offspring: implications in the development of the cardiorenal metabolic syndrome in offspring. Cardiorenal Med. 2012:2:134-42.

11. Farrar D, Simmonds M, Bryant M, Sheldon TA, Tuffnell D, Golder S, et al. Hyperglycaemia and risk of adverse perinatal outcomes: systematic review and meta-analysis. BMJ. 2016:354:i4694.

12. Farrar D, Simmonds M, Bryant M, Sheldon TA, Tuffnell D, Golder S, et al. Treatments for gestational diabetes: a systematic review and meta-analysis. BMJ Open. 2017;7:e015557.

13. Poolsup N, Suksomboon N, Amin M. Effect of treatment of gestational diabetes mellitus: a systematic review and meta-analysis. PLoS ONE. 2014:9:e92485.
14. Crowther CA, Hiller JE Moss JR, MCPhee AJ, Jeffries WS, Robinson JS Effect of treatment of gestational diabetes mellitus on pregnancy outcomes. N Engl J Med. 2005;352:2477-86.

15. Farrar D, Duley L, Dowswell T, Lawlor DA. Different strategies for diagnosing gestational diabetes to improve maternal and infant health. Cochrane Database Syst Rev. 2017;8:CD007122.

16. Carreiro M, Nogueira A, Ribeiro-Oliveira A. Controversies and advances in gestational diabetes - an update in the era of continuous glucose monitoring. J Clin Med. 2018. https://doi.org/10.3390/jcm7020011.

17. Hosseini $\mathrm{E}$, Janghorbani M, Aminorroaya A. Incidence, risk factors, and pregnancy outcomes of gestational diabetes mellitus using one-step versus two-step diagnostic approaches: a population-based cohort study in Isfahan, Iran. Diabetes Res Clin Pract. 2018;140:288-94.

18. Landon MB, Rice MM, Varner MW, Casey BM, Reddy UM, Wapner RJ, et al. Mild gestational diabetes mellitus and long-term child health. Diabetes Care. 2015:38:445-52

19. Landon MB. Is there a benefit to the treatment of mild gestational diabetes mellitus? Am J Obstet Gynecol. 2010;202:649-53.

20. Ohno MS, Sparks TN, Cheng YW, Caughey AB. Treating mild gestational diabetes mellitus: a cost-effectiveness analysis. Am J Obstet Gynecol. 2011;205(282):e1-7.

21. Rice MM, Landon MB, Units HDMFM, Health EKSNIoC. What we have learned about treating mild gestational diabetes mellitus. Semin Perinatol. 2016;40:298-302.

22. Diabetes IAo, Panel PSGC. International association of diabetes and pregnancy study groups recommendations on the diagnosis and classification of hyperglycemia in pregnancy. Diabetes Care. 2010;33:676-82

23. Olagbuji BN, Atiba AS, Olofinbiyi BA, Akintayo AA, Awoleke JO, Ade-Ojo IP, et al. Prevalence of and risk factors for gestational diabetes using 1999, 2013 WHO and IADPSG criteria upon implementation of a universal one-step screening and diagnostic strategy in a sub-Saharan African population. Eur J Obstet Gynecol Reprod Biol. 2015;189:27-32.

24. Kanguru L, Bezawada N, Hussein J, Bell J. The burden of diabetes mellitus during pregnancy in low-and middle-income countries: a systematic review. Glob Health Action. 2014;7:23987.

25. Mayo K, Melamed N, Vandenberghe $\mathrm{H}$, Berger $\mathrm{H}$. The impact of adoption of the international association of diabetes in pregnancy study group criteria for the screening and diagnosis of gestational diabetes. Am J Obstet Gynecol. 2015;212(224):e1-9.

26. Fitria N, van Asselt AD, Postma MJ. Cost-effectiveness of controlling gestational diabetes mellitus: a systematic review. Eur J Health Econ. 2019:20:407-17.

27. Marchetti D, Carrozzino D, Fraticelli F, Fulcheri M, Vitacolonna E. Quality of life in women with gestational diabetes mellitus: a systematic review. J Diabetes Res. 2017;2017:7058082

28. Kalra B, Gupta Y, Baruah MP. Renaming gestational diabetes mellitus: a psychosocial argument. Indian J Endocrinol Metab. 2013;17:S593-5.

29. Kalra S, Baruah MP, Gupta Y, Kalra B. Gestational diabetes: an onomastic opportunity. Lancet Diabetes Endocrinol. 2013;1:91.

30. Poncet B, Touzet S, Rocher L, Berland M, Orgiazzi J, Colin C. Cost-effectiveness analysis of gestational diabetes mellitus screening in France. Eur J Obstet Gynecol Reprod Biol. 2002;103:122-9.

31. Diagnostic criteria and classification of hyperglycaemia first detected in pregnancy. World Health Organization report 2013. http://apps.who. int/iris/bitstream/handle/10665/85975/WHO_NMH_MND_13.2_eng. pdf;jsessionid=F503D782279A637B1B1D7AC70CC88404? sequence $=1$.

32. Lohse N, Marseille E, Kahn JG. Development of a model to assess the cost-effectiveness of gestational diabetes mellitus screening and lifestyle change for the prevention of type 2 diabetes mellitus. Int J Gynaecol Obstet. 2011:115:S20-5.

33. Round J, Jacklin P, Fraser R, Hughes R, Mugglestone M, Holt R. Screening for gestational diabetes mellitus: cost-utility of different screening strategies based on a woman's individual risk of disease. Diabetologia. 2011:54:256-63.

34. Oostdam N, Bosmans J, Wouters MG, Eekhoff EM, van Mechelen W, van Poppel MN. Cost-effectiveness of an exercise program during pregnancy to prevent gestational diabetes: results of an economic evaluation alongside a randomised controlled trial. BMC Pregnancy Childbirth. 2012;12:64.

35. Farrar D, Simmonds M, Griffin S, Duarte A, Lawlor DA, Sculpher M, et al. The identification and treatment of women with hyperglycaemia in pregnancy: an analysis of individual participant data, systematic reviews, 
meta-analyses and an economic evaluation. Health Technol Assess. 2016;20:1-348.

36. Kolu P, Raitanen J, Puhkala J, Tuominen P, Husu P, Luoto R. Effectiveness and cost-effectiveness of a cluster-randomized prenatal lifestyle counseling trial: a seven-year follow-up. PLoS ONE. 2016;11:e0167759.

37. Moss JR, Crowther CA, Hiller JE, Willson KJ, Robinson JS. Costs and consequences of treatment for mild gestational diabetes mellitus - evaluation from the ACHOIS randomised trial. BMC Pregnancy Childbirth. 2007;7:27.

38. O'Dea A, Infanti JJ, Gillespie P, Tummon O, Fanous S, Glynn LG, et al. Screening uptake rates and the clinical and cost effectiveness of screening for gestational diabetes mellitus in primary versus secondary care: study protocol for a randomised controlled trial. Trials. 2014;15:27.

39. Drummond MF, Sculpher MJ, Torrance GW, O'Brien BJ, Stoddart GL. Methods for the economic evaluation of health care programmes. Oxford: Oxford University Press; 2005.

40. Weile LK, Kahn JG, Marseille E, Jensen DM, Damm P, Lohse N. Global costeffectiveness of GDM screening and management: current knowledge and future needs. Best Pract Res Clin Obstet Gynaecol. 2015;29:206-24.

41. https://www.buckeyehealthplan.com/content/dam/centene/Buckeye/ medicaid/pdfs/ACOG-Guidelines-for-Perinatal-Care.pdf

42. Piaggio G, Elbourne DR, Pocock SJ, Evans SJ, Altman DG, CONSORT Group $\mathrm{ft}$. Reporting of noninferiority and equivalence randomized trials: extension of the CONSORT 2010 statement. JAMA. 2010;2012(308):2594-604.

43. Gestational Diabetes. Clinical, management guildline. http://aegleclini c.com/wp-content/uploads/2015/05/Gestational-Diabetes-ACOG-2013. pdf.

44. Standards of Medical Care in Diabetes-2016. Diabetes Care. 2016;39:S4-5.

45. Montazeri A, Goshtasebi A, Vahdaninia M, Gandek B. The Short Form Health Survey (SF-36): translation and validation study of the Iranian version. Qual Life Res. 2005:14:875-82.

46. Montazeri A, Vahdaninia M, Mousavi SJ, Omidvari S. The Iranian version of 12-item Short Form Health Survey (SF-12): factor structure, internal consistency and construct validity. BMC Public Health. 2009;9:341.

47. Darvishpoor Kakhki A, Abed Saeedi Z. Health-related quality of life of diabetic patients in Tehran. Int J Endocrinol Metab. 2013;11:e7945.

48. Kiadaliri AA, Najafi B, Mirmalek-Sani M. Quality of life in people with diabetes: a systematic review of studies in Iran. J Diabetes Metab Disord. 2013;12:54

49. American College of Obstetricians and Gynecologists' Committee on Practice Bulletins-Obstetrics. Practice Bulletin No. 173: Fetal Macrosomia. Obstet Gynecol. 2016;128:e195-209.

50. Boyle A, Reddy U, Landy H, Huang C, Driggers R, Laughon S. Primary cesarean delivery in the United States. Obstet Gynecol. 2013;1 22:33-40.

51. Aviram A, Salzer L, Hiersch L, Ashwal E, Golan G, Pardo J, et al. Association of isolated polyhydramnios at or beyond 34 weeks of gestation and pregnancy outcome. Obstet Gynecol. 2015;125:825-32.

52. Kim BJ, Romero R, Lee SM, Park C-W, Park JS, Jun JK, et al. Clinical significance of oligohydramnios in patients with preterm labor and intact membranes. J Perinat Med. 2011;39:131-6.
53. American College of Obstetricians and Gynecologists' Committee on Practice Bulletins-Obstetrics. Practice Bulletin No. 24: Fetal Growth Restriction. Obstet Gynecol. 2013;133:e97-108.

54. Gardosi J, Clausson B, Francis A. The value of customised centiles in assessing perinatal mortality risk associated with parity and maternal size. BJOG. 2009;116:1356-63.

55. Sweet CB, Grayson S, Polak M. Management strategies for neonatal hypoglycemia. J Pediatr Pharmacol Ther. 2013;18:199-208.

56. Cornblath M, Hawdon JM, Williams AF, Aynsley-Green A, Ward-Platt MP, Schwartz R, et al. Controversies regarding definition of neonatal hypoglycemia: suggested operational thresholds. Pediatrics. 2000;105:1141-5.

57. Maisels MJ, Bhutani VK, Bogen D, Newman TB, Stark AR, Watchko JF. Hyperbilirubinemia in the newborn infant $\geq 35$ weeks' gestation: an update with clarifications. Pediatrics. 2009;124:1193-8.

58. Mustafa R, Ahmed S, Gupta A, Venuto RC. A comprehensive review of hypertension in pregnancy. J Pregnancy. 2012;2012:105918.

59. https://www.acog.org/ /media/Task\%20Force\%20and\%20Work\%20Gro up\%20Reports/public/HypertensioninPregnancy.pdf.

60. Committee on Practice Bulletins-Obstetrics T. Practice bulletin no. 130: prediction and prevention of preterm birth. Obstet Gynecol. 2012;120:964-73

61. Chauhan SP, Gherman R, Hendrix NW, Bingham JM, Hayes E. Shoulder dystocia: comparison of the ACOG practice bulletin with another national guideline. Am J Perinatol. 2010;27:129-36.

62. Dean RB, Dixon W. Simplified statistics for small numbers of observations. Anal Chem. 1951;23:636-8.

63. Lin L, Sherman PD. Cleaning data the Chauvenet way. In: The Proceedings of the SouthEast SAS Users Group, SESUG Proceedings, Paper SA11. 2007.

64. Basu A, Rathouz PJ. Estimating marginal and incremental effects on health outcomes using flexible link and variance function models. Biostatistics. 2005:6:93-109.

65. Briggs AH. A Bayesian approach to stochastic cost-effectiveness analysis. Health Econ. 1999:8(3):257-61.

66. Briggs A, Bousquet J, Wallace M, Busse WW, ClarkT, Pedersen S, et al. Cost-effectiveness of asthma control: an economic appraisal of the GOAL study. Allergy. 2006;61:531-6.

67. Briggs A, Fenn P. Confidence intervals or surfaces? Uncertainty on the cost-effectiveness plane. Health Econ. 1998;7:723-40.

68. Schaefer AJ, Bailey MD, Shechter SM, Roberts MS. Modeling medical treatment using Markov decision processes. In: Brandeau ML, Sainfort F, Pierskalla WP, editors. Operations research and health care. Springer: Boston; 2005. p. 593-612

69. Komorowski M, Raffa J. Markov models and cost effectiveness analysis: applications in medical research. In: Secondary analysis of electronic health records. Cham: Springer; 2016. p. 351-67.

\section{Publisher's Note}

Springer Nature remains neutral with regard to jurisdictional claims in published maps and institutional affiliations.
Ready to submit your research? Choose BMC and benefit from:

- fast, convenient online submission

- thorough peer review by experienced researchers in your field

- rapid publication on acceptance

- support for research data, including large and complex data types

- gold Open Access which fosters wider collaboration and increased citations

- maximum visibility for your research: over $100 \mathrm{M}$ website views per year

At BMC, research is always in progress.

Learn more biomedcentral.com/submissions 